Production, Manufacturing and Logistics

\title{
Replenishment decisions under an all-units discount schedule and stepwise freight costs
}

\author{
Ayşegül Toptal * \\ Industrial Engineering Department, Bilkent University, 06800 Ankara, Turkey
}

\section{A R T I C L E I N F O}

\section{Article history:}

Received 14 February 2007

Accepted 1 September 2008

Available online 10 October 2008

\section{Keywords:}

All-units discount

Transportation

Cargo capacity

Newsboy model/problem

\begin{abstract}
A B S T R A C T
In this study, we analyze the replenishment decisions under a general replenishment cost structure that includes stepwise freight costs and all-units quantity discounts. We first formulate a general model that accounts for a larger class of problems and prove several useful properties of the expected profit function. We later utilize these properties to develop a computational solution approach to find the optimal order quantity. As an application of the general results, we study the replenishment decisions in the single-period, i.e., the Newsboy, problem considering several scenarios that model the cost considerations either for the buyer or for both the buyer and the vendor.
\end{abstract}

(c) 2008 Elsevier B.V. All rights reserved.

\section{Introduction and literature}

Transportation is a significant activity of supply chain operations, and there is ample evidence that the consideration of transportation with inventory replenishment decisions can lower the total costs. This can bring competitive advantage to companies, specifically to those with functional products and for which minimization of costs is a major priority. Recognizing this benefit, several studies in single echelon lot sizing literature in the 1980s and the early 1990s accounted for transportation costs $[3,18,25]$. This issue has recently regained attention in supply chain research, due to an increasing trend in practice to outsource logistical activities through third party companies. The current study focuses on the ordering decisions of a company that faces a generalized wholesale price schedule from a supplier(s) and stepwise freight costs charged by a third party logistic (3PL) company for inbound replenishment.

As reported by Benton and Park [5], offering quantity discounts to encourage buyers to order more is a common pricing strategy of suppliers. Traditionally, the models on quantity discounts either take the buyer's point of view or the supplier's point view. The former group focus on the buyer's problem to decide his/her replenishment quantity under a given quantity discount schedule $[1,2,11,26]$. Others demonstrate that through a carefully designed price schedule, a vendor can increase gain $[4,17,20,21,29]$. In this latter group of studies, a common assumption is that the vendor has full information about the buyer's costs. In a more recent study, Corbett and de Groote [10] consider a supplier's optimal quantity

\footnotetext{
* Tel.: +90 3122901702.

E-mail address: toptal@bilkent.edu.tr.
}

discount policy under asymmetric information in a single-buyer, single-supplier, deterministic demand setting. Munson and Rosenblatt [22] provide an extensive review of the literature on quantity discounts until the late 1990s. The current study follows the former group of studies and is aimed at developing a computational solution approach to find the optimal order quantity that maximizes a buyer's single-period expected profits under an all-units discount schedule and stepwise freight costs.

All-units discounts and incremental discounts are the two discounting schemes that are most commonly seen in the industry and investigated in the literature [22]. In fact, these structures are not only used for wholesale pricing by vendors, but they are also adopted by common carriers, e.g., see $[16,23,25]$. Quantity discount schedules, when they are used for transportation pricing, are regarded as belonging within the class of LTL (less-than-truck-load) transportation pricing. In another form of freight cost structure, i.e., TL transportation, a fixed amount is charged for each additional truck/container deployed. Aucamp [3] studies a special case in which the per-truck cost is constant, independent of the order quantity. Lee [18] generalizes Aucamp's [3] work to consider discounted per-truck costs for larger replenishment quantities. Shinn et al. [24] study the lot sizing and pricing problems jointly for a retailer under conditions of discounted freight costs and permissible delay in payments. Lee [19] incorporates the transportation cost structure in Aucamp [3] to the classical dynamic lot size model.

In the current study, assuming the same freight cost structure as in Aucamp [3], we generalize the replenishment costs further, by modeling the wholesale price $c(Q)$ according to an all-units discount schedule with multiple breakpoints. More specifically, the sum of procurement and replenishment costs of the buyer for ordering $Q$ units is given by 
$C(Q)=c(Q) Q+\left\lceil\frac{Q}{P}\right\rceil R$,

where $P$ and $R$ are the per-truck capacity and per-truck cost, respectively. The cost value $R$ is incurred for each truck, whether it is fully or partially loaded.

Transportation costs have been previously considered in single echelon lot sizing problems involving quantity discounts in purchasing price, e.g., see $[6,25]$. However, freight costs in these studies are modeled in the form of LTL transportation pricing. A similar problem to ours has been studied by Hwang et al. [15]. While they study the replenishment problem under similar cost considerations to ours, their model is based on the specific assumptions of the classical economic order quantity (EOQ) model. In the current study, however, the production/inventory related net profits are modeled using a general function exhibiting some structural properties. This allows for a technical representation of various replenishment problems in different settings, including those with random demand and/or multiple echelons, as discussed in Section 4. Furthermore, we provide additional insights regarding the application areas of our model and discuss some managerial implications.

It is worth noting that the freight cost structure as in the second term of Expression (1) has also been used to model integrated inventory/transportation decisions in multi-stage inventory systems $[8,27,28]$. Other studies on the computation of jointly optimal order quantities in multi-echelon systems under transportation considerations include Chan et al. [9], Hoque and Goyal [14], and Ertogral et al. [12].

All of the studies discussed above within the context of quantity discounts and transportation considerations assume deterministic demand, with the exception of Toptal and Cetinkaya [28]. In this paper, the authors study the channel coordination problem of a buyer-vendor system in the Newsboy setting under the assumption that either the vendor or both the buyer and the vendor face transportation costs as introduced in Expression (1). In their analysis, they present the characteristics of the optimal solution maximizing a function that includes a concave component reduced by stepwise fixed increments. Some of our analysis will use the analytical results provided in Toptal and Çetinkaya [28].

The underlying assumptions regarding the buyer's inventory policy and cost components in this study are general, except for the wholesale price structure and the transportation considerations. In Section 2, we present a generic mathematical formulation that can be used to solve a large class of problems under the given assumptions. An analysis for this model with the description of the proposed computational solution approach will follow in Section 3. The application of the model to various scenarios within the Newsboy setting will be discussed in Section 4, and the implications for inventory management will be explained in Section 5. The paper will be concluded in Section 6 with a summary of the general findings.

\section{Notation and problem formulation}

Let us consider a retailer who has to make replenishment decisions under an all-units discount schedule. Specifically, the unit wholesale price, denoted by $c(Q)$ is given by the following expression:

$c(Q)= \begin{cases}c_{0} & q_{0} \leqslant Q<q_{1}, \\ c_{1} & q_{1} \leqslant Q<q_{2}, \\ c_{2} & q_{2} \leqslant Q<q_{3}, \\ \vdots & \vdots \\ c_{n-1} & q_{n-1} \leqslant Q<q_{n}, \\ c_{n} & Q \geqslant q_{n},\end{cases}$

where $q_{0}=0$ and $c_{0}>c_{1}>\ldots>c_{n}$.
The retailer has to pay for his/her transportation costs, which amount to $\left[\frac{Q}{P}\right] R$ for an order quantity of $Q$. Here, $P$ and $R$ are the per-truck capacity and the per-truck cost, respectively. The profit function of the retailer is then given by

$\mathscr{H}(Q)=G(Q, c(Q))-\left\lceil\frac{Q}{P}\right\rceil R$

where $G\left(Q, c_{i}\right)$ is a strictly concave function of $Q, \forall i$ s.t. $0 \leqslant i \leqslant n$. The function $G\left(Q, c_{i}\right)$ may represent the production/inventory related net profits of the retailer. Let the unique maximizer of this function over $Q \geqslant 0$ be denoted by $Q^{(i)}$. We say $Q^{(i)}$ is realizable if $q_{i} \leqslant Q^{(i)}<q_{i+1}$ and call the maximum of all the realizable $Q^{(i)}$ s over $0 \leqslant i \leqslant n$ the largest realizable maximizer of $G\left(Q, c_{i}\right)$ s and refer to it as $Q^{\left(r_{2}\right)}$. We have the following assumptions:

(A1) The maximizer of $G\left(Q, c_{i}\right)$ increases as $c_{i}$ decreases. That is, we have

$Q^{(n)}>Q^{(n-1)}>\ldots>Q^{(0)}$.

(A2) For a fixed value of $Q, G\left(Q, c_{i}\right)$ is decreasing in $c_{i}$. That is, we have

$G\left(Q, c_{0}\right)<G\left(Q, c_{1}\right)<\ldots<G\left(Q, c_{n}\right)$.

(A3) The change in the $G\left(Q, c_{i}\right)$ value when $c_{i}$ is decreased, increases with respect to $Q$. More specifically,

$G\left(Q_{2}, c_{i+1}\right)-G\left(Q_{2}, c_{i}\right)>G\left(Q_{1}, c_{i+1}\right)-G\left(Q_{1}, c_{i}\right)$,

where $Q_{1}<Q_{2} \leqslant Q^{(i)}$.

Under the above assumptions, the retailer decides on his/her replenishment quantity according to the formulation given by Problem $\mathscr{D} \mathscr{P} \mathscr{T} \mathscr{C}$ (discounted price transportation cost),

$\mathscr{D P} \mathscr{T} \mathscr{C}:$

$\max \mathscr{H}(Q)$,

s.t. $Q \geqslant 0$.

Let $Q^{*}$ denote the solution of this problem. Note that $\mathscr{H}(Q)$ is composed of $(n+1)$ pieces with the function value on the $(i+1)$ st piece given by $H^{i}(Q)$, where

$H^{i}(Q)=G\left(Q, c_{i}\right)-\left\lceil\frac{Q}{P}\right\rceil R$.

Assume that $\tilde{Q}^{(i)}$ maximizes $H^{i}(Q)$ over $Q \geqslant 0$. We say $\tilde{Q}^{(i)}$ is realizable if $q_{i} \leqslant \tilde{Q}^{(i)}<q_{i+1}$ and call the maximum of all the realizable $\tilde{Q}^{(i)}$ s over $0 \leqslant i \leqslant n$ the largest realizable maximizer of $H^{i}(Q)$ s and refer to it as $\tilde{Q}^{\left(r_{1}\right)}$.

The notation used in the paper is as follows:

$Q \quad$ number of items ordered by the retailer

$n \quad$ number of price breakpoints

$q_{i} \quad$ quantity where the $i$ th breakpoint appears, $0 \leqslant i \leqslant n$

$c(Q) \quad$ unit wholesale price as a function of order quantity

$P \quad$ per-truck capacity

$R \quad$ per-truck cost

$\mathscr{H}(Q) \quad$ profit function of the retailer

$H^{i}(Q) \quad$ profit function of the retailer at wholesale price level $c_{i}$, defined over $Q \geqslant 0$

$G\left(Q, c_{i}\right)$ retailer's profit component defined over $Q \geqslant 0$ for price level $c_{i}$, not including transportation costs

$Q^{*} \quad$ maximizer of $\mathscr{H}(Q)$

$Q^{(i)} \quad$ maximizer of $G\left(Q, c_{i}\right)$

$\tilde{Q}^{(i)} \quad$ maximizer of $H^{i}(Q)$

$r_{1} \quad$ index of the price interval where the largest realizable maximizer of $H^{i}(Q)$ s appears: $r_{1}=\max \left\{i: q_{i} \leqslant \tilde{Q}^{(i)}<\right.$ $q_{i+1}$, and $\left.0 \leqslant i \leqslant n\right\}$

$r_{2} \quad$ index of the price interval where the largest realizable maximizer of $G\left(Q, c_{i}\right)$ s appears: $r_{2}=\max \left\{i: q_{i} \leqslant Q^{(i)}<\right.$ $q_{i+1}$, and $\left.0 \leqslant i \leqslant n\right\}$ 
The model introduced above is general in the sense that the $G\left(Q, c_{i}\right)$ function may represent the expected profits in several production/procurement environments. In the next section, by analyzing the properties of the underlying profit functions first, specifically those of $G\left(Q, c_{i}\right)$ and $H^{i}(Q)$, we present an algorithm to solve Problem $\mathscr{D P} \mathscr{T} \mathscr{C}$. This solution will then be applied to the Newsboy setting.

\section{Analysis of the problem}

In this section, we provide a computational solution approach to solving Problem $\mathscr{D P} \mathscr{T} \mathscr{C}$ optimally. As seen in Fig. $1, H(Q)$, the objective function of this problem, has a piecewise structure. In order to find its maximizer $Q^{*}$, we will first analyze some structural properties of the $H^{i}(Q)$ function and the solution to the following problem, referred to as Problem $\mathscr{U} \mathscr{P} \mathscr{T} \mathscr{C}$ (uniform price transportation cost):

$\mathscr{U} \mathscr{P} \mathscr{T} \mathscr{C}:$

$\max H^{i}(Q)$,

s.t. $Q \geqslant 0$.

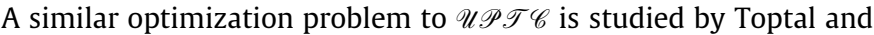
Çetinkaya [28] for Expression (4) with the concave functional form of $G\left(Q, c_{i}\right)$. Properties $1-5$, which will be presented below without their proofs, are based on the analysis in Toptal and Çetinkaya and are straightforward to verify.

Property 1. Let $Q_{2}>Q_{1}>Q^{(i)}$. Then $H^{i}\left(Q_{2}\right)<H^{i}\left(Q_{1}\right)$. That is, $H^{i}(Q)$ is decreasing after $Q^{(i)}$.

Property 1 implies that the solution to Problem $\mathscr{U P \mathscr { T } \mathscr { C }}$ lies in the region $\left[0, Q^{(i)}\right]$. Before presenting further properties of the $H^{i}(Q)$ function, let us define $l$ as the smallest number of full truck loads greater than or equal to $Q^{(i)}$. That is, $l=\left\lceil\frac{Q^{(i)}}{P}\right\rceil$.

Property 2. Let $Q_{1}$ and $Q_{2}$ be such that $(k-1) P<Q_{1}<$ $Q_{2} \leqslant k P<Q^{(i)}$ where $k \geqslant 1$, or $(l-1) P<Q_{1}<Q_{2} \leqslant Q^{(i)}$. Then, $H^{i}\left(Q_{1}\right)<H^{i}\left(Q_{2}\right)$. In other words, for $Q \leqslant Q^{(i)}, H^{i}(Q)$ is piecewise increasing.

It follows from Property 2 that the only candidates for $\tilde{Q}^{(i)}$ within $\left[0, Q^{(i)}\right]$ are the largest quantities of each piecewise interval.
Property 3. Let us define

$$
\begin{aligned}
& \begin{aligned}
\mathscr{F} & =\left\{k \in\{0,1,2, \ldots\}: G\left((k+1) P, c_{i}\right)-G\left(k P, c_{i}\right) \leqslant R,(k+1) P\right. \\
& \left.\leqslant Q^{(i)}\right\} .
\end{aligned} \\
& \text { If } \mathscr{F} \neq \emptyset \text {, let } m=\min \{k \text { s.t. } k \in \mathscr{F}\} . \text { It follows that }
\end{aligned}
$$

- If $G\left((m+1) P, c_{i}\right)-G\left(m P, c_{i}\right)=R$, then $H^{i}((j+1) P)<H^{i}(j P), \forall j$ s.t. $j \geqslant(m+1)$ and $(j+1) P \leqslant Q^{(i)}$.

- If $G\left((m+1) P, c_{i}\right)-G\left(m P, c_{i}\right)<R$, then $H^{i}((j+1) P)<H^{i}(j P), \forall j$ s.t. $j \geqslant m$ and $(j+1) P \leqslant Q^{(i)}$.

That is, in the region $Q \leqslant Q^{(i)}$, the value of $G\left(Q, c_{i}\right)$ at integer multiples of $P$ starts to decrease after $(m+1) P$ in the first case and after $m P$ in the second case.

Property 3 characterizes the conditions under which the cost of an additional truck does not justify the benefits of ordering more. If these conditions are satisfied, there exists a quantity until which ordering one more full truck load is always profitable. Therefore, in solving Problem $\mathscr{U} \mathscr{P} \mathscr{T} \mathscr{C}$, all order sizes that are smaller than this quantity can be eliminated. Properties 4 and 5 build on Property 3 to provide further characteristics of $\tilde{Q}^{(i)}$.

Property 4. If $\mathscr{F}=\emptyset$, then either $(l-1) P$ or $Q^{(i)}$, or both maximize $H^{i}(Q)$.

Property 5. If $\mathscr{F} \neq \emptyset$, then either $m P$ or $(m+1) P$, or both maximize $H^{i}(Q)$.

The above properties of $H^{i}(Q)$ lead to the following solution for Problem $\mathscr{U} \mathscr{P} \mathscr{T} \mathscr{C}$.

Corollary 1. As a result of Properties 1 to 5 , the solution to Problem $\mathscr{U P \mathscr { T } \mathscr { C }}$ is given by

$$
\tilde{Q}^{(i)}= \begin{cases}\arg \max \left\{H^{i}(m P), H^{i}((m+1) P)\right\} & \text { if } \mathscr{F} \neq \emptyset, \\ \arg \max \left\{H^{i}\left(Q^{(i)}\right), H^{i}((l-1) P)\right\} & \text { if } \mathscr{F}=\emptyset,\end{cases}
$$

where

$$
\mathscr{F}=\left\{k \in\{0,1,2, \ldots\}: G\left((k+1) P, c_{i}\right)-G\left(k P, c_{i}\right) \leqslant R,(k+1) P \leqslant Q^{(i)}\right\}
$$
and $m=\min \{k$ s.t. $k \in \mathscr{F}\}$ when $\mathscr{F} \neq \emptyset$.

Note that, under both conditions of the corollary, multiple solutions may exist. In the first case, if $G\left((m+1) P, c_{i}\right)-G\left(m P, c_{i}\right)<R$, then $m P$ is the unique maximizer. If $G\left((m+1) P, c_{i}\right)-$

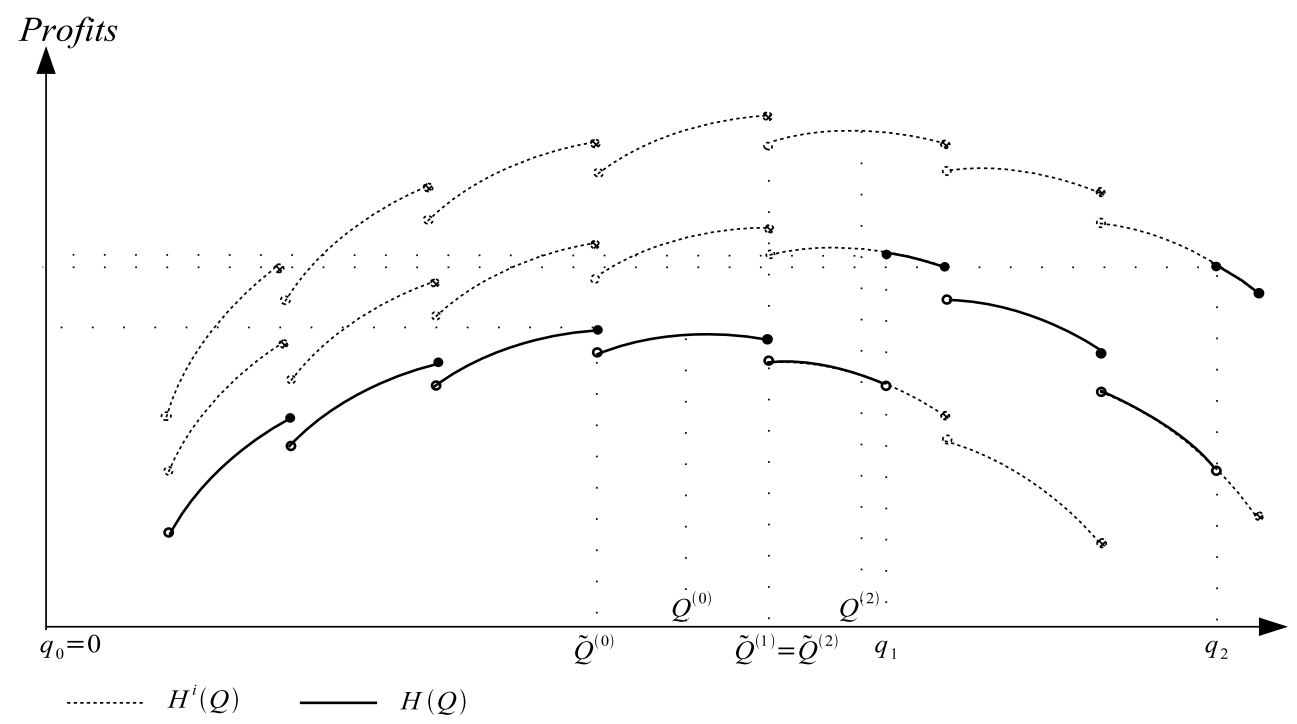

Fig. 1. A typical illustration of $H(Q)$ in the case of two breakpoints. 
$G\left(m P, c_{i}\right)=R$, then both $m P$ and $(m+1) P$ maximize $H^{i}(Q)$. Similarly, in the second case, if $G\left(Q^{(i)}, c_{i}\right)-G\left((l-1) P, c_{i}\right)<R$, then $Q^{(i)}$ is the unique maximizer. If $G\left(Q^{(i)}, c_{i}\right)-G\left((l-1) P, c_{i}\right)=R$, then both $Q^{(i)}$ and $(l-1) P$ maximize $H^{i}(Q)$.

Observe that the objective function of Problem $\mathscr{D P} \mathscr{T} \mathscr{C}$, i.e., $H(Q)$, is composed of $(n+1)$ different pieces in the form of $H^{i}(Q)$, given by different values of $c_{i}$. Based on the above properties of an $H^{i}(Q)$ function and the solution provided in Corollary 1 to maximize it, we next focus on the more complex structure exhibited by $H(Q)$. The proofs of Properties 6-10, Corollary 3, and Propositions 1,2 are presented in Appendix.

Property 6. Let $Q_{1}$ and $Q_{2}$ be such that $q_{k-1} \leqslant Q_{2}<Q_{1}<q_{k}$ where $k>\left(r_{2}+1\right)$. It follows that $G\left(Q_{1}, c_{k-1}\right)<G\left(Q_{2}, c_{k-1}\right)$. That is, $G\left(Q, c_{k-1}\right)$ is decreasing over $q_{k-1} \leqslant Q<q_{k}$ for all $k$ larger than $r_{2}+1$.

Property 6 states that when the stepwise component of Expression (3) is ignored, the remaining part, i.e., $G(Q, c(Q))$, is piecewise decreasing after the price interval, where the largest realizable maximizer of $G\left(Q, c_{i}\right)$ s appears.

Corollary 2. We have $Q^{(k)}<q_{k}, \forall k$ s.t. $k \geqslant\left(r_{2}+1\right)$.

Proof. Follows from Property 6 and the definition of $r_{2}$.

Property 7. $\mathscr{H}\left(q_{k-1}\right)>\mathscr{H}(Q)$ where $q_{k-1}<Q<q_{k}, \forall k$ s.t. $k>r_{2}+1$.

Property 7 implies that in maximizing $\mathscr{H}(Q)$, among all quantities greater than $Q^{\left(r_{2}\right)}$, we should only consider the breakpoints $q_{r_{2}+1}, \ldots, q_{n}$.

Property 8. $H^{i}\left(\tilde{Q}^{(i)}\right)<H^{i+1}\left(\tilde{Q}^{(i+1)}\right), \forall i$ s.t. $0 \leqslant i \leqslant n-1$. That is, the optimal function values at consecutive $H^{i}(Q)$ s are increasing.

Note that Property 8 implies $H^{n}\left(\tilde{Q}^{(n)}\right)>$ $H^{n-1}\left(\tilde{Q}^{(n-1)}\right)>\ldots>H^{0}\left(\tilde{Q}^{(0)}\right)$. Therefore, if the maximizer of $H^{i}(Q)$ is realizable, then $\mathscr{H}\left(\tilde{Q}^{(i)}\right) \geqslant \mathscr{H}(Q), \forall Q<q_{i+1}$. This further leads to the fact that, in maximizing $\mathscr{H}(Q)$, we do not need to consider quantities smaller than the largest realizable maximizer of $H^{i}(Q)$ s.

Property 9. We have $\tilde{Q}^{(i)} \leqslant \tilde{Q}^{(i+1)}, \forall i$ s.t. $0 \leqslant i \leqslant n-1$. In other words, the maximizers of consecutive $H^{i}(Q)$ functions are nondecreasing.

Next, Property 9 will be used to prove that there exists at least one realizable $\tilde{Q}^{(i)}$, and hence, $r_{1}$ exists.

Property 10. There exists $i \in\{0,1, \ldots, n\}$ such that $q_{i} \leqslant \tilde{Q}^{(i)}<q_{i+1}$.

Note that the solution to the classical economic order quantity model with all-units quantity discounts builds on the fact that there is at least one realizable EOQ (see Hadley and Whitin [13]). With a similar proof as that of Property 10, it is easy to show that the same result holds for the Newsboy Model with all-units quantity discounts. That is, there exists $i \in\{0,1, \ldots, n\}$ such that $q_{i} \leqslant Q^{(i)}<q_{i+1}$, and hence, $r_{2}$ exists. The following corollary presents a relationship between $r_{1}$ and $r_{2}$.

Corollary 3. We have $r_{1} \leqslant r_{2}$ and $\tilde{Q}^{\left(r_{1}\right)} \leqslant Q^{\left(r_{2}\right)}$.

Properties 7, 8, 10 and Corollary 3 provide the main results leading to the solution algorithm that will be introduced in Corollary 4 . The proposition that will be presented next builds mainly on Corollaries 1 and 3 and will later be used to enhance Corollary 4.

Proposition 1. If $r_{2}>r_{1}+1$, then we have $\tilde{Q}^{(k)}<q_{k}, \forall k$ s.t. $r_{1}<k<r_{2}$.

Recall from Corollary 1 that, we have $\tilde{Q}^{(i)} \leqslant Q^{(i)}, \forall i$ s.t. $0 \leqslant i \leqslant n$. If $r_{2}>r_{1}+1$, this implies that $\tilde{Q}^{\left(r_{2}\right)}<q_{r_{2}}$. Therefore, solving Problem $\mathscr{D} \mathscr{P} \mathscr{T} \mathscr{C}$ involves minimizing $H^{i}(Q)$ functions over $q_{i} \leqslant Q<q_{i+1}$ for $i \in\left[r_{1}+1, r_{2}\right]$, where we already know that $\tilde{Q}^{(i)}<q_{i}$. Combining this result with Properties 7 and 8, we present an algorithm in the next corollary to solve Problem $\mathscr{D} \mathscr{P} \mathscr{T} \mathscr{C}$ based on the values of $r_{1}$ and $r_{2}$.

Corollary 4. Given the values of $r_{1}, r_{2}$, and $\tilde{Q}^{\left(r_{1}\right)}$, the following algorithm solves Problem $\mathscr{D} \mathscr{P} \mathscr{T} \mathscr{C}$ optimally:

1. Set $Q^{*}=\tilde{Q}^{\left(r_{1}\right)}$ and compute $\mathscr{H}\left(Q^{*}\right)$.

2. If $r_{1}=r_{2}$ go to Step 4 , else proceed with the next step.

3. For $i=r_{1}+1$ to $i=r_{2}$ do the following.

(a) Solve Problem $\mathscr{U P \mathscr { B } \mathscr { I }}$ (uniform price bounded interval) where

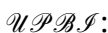

$\max H^{i}(Q)$,

s.t. $\quad q_{i} \leqslant Q<q_{i+1}$.

Let the optimal solution to the above problem be $Q_{i}^{*}$.

(b) Compute $\mathscr{H}\left(Q_{i}^{*}\right)$. If $\mathscr{H}\left(Q_{i}^{*}\right)>\mathscr{H}\left(Q^{*}\right)$, let $Q^{*}=Q_{i}^{*}$.

4. If $r_{2}<n$, compute $q_{\text {max }}=\arg \max \left\{\mathscr{H}\left(q_{r_{2}+1}\right), \mathscr{H}\left(q_{r_{2}+2}\right), \ldots\right.$, $\left.\mathscr{H}\left(q_{n}\right)\right\}$. If $\mathscr{H}\left(q_{\max }\right)>\mathscr{H}\left(Q^{*}\right)$, let $Q^{*}=q_{\max }$.

It is important to emphasize that since $Q^{(n)}>Q^{(n-1)}>\cdots>Q^{(0)}$, we should start from the lowest price interval first to find the value of $r_{2}$. This requires computing the $Q^{(i)}$ value for the corresponding $G\left(Q, c_{i}\right)$ function and checking whether $q_{i} \leqslant Q^{(i)}<q_{i+1}$. We know from Corollary 3 that $r_{1} \leqslant r_{2}$. Combining this result with Property 9 , we further conclude that once the value of $r_{2}$ is fixed, one should check the remaining price intervals starting from $\left[q_{r_{2}}, q_{r_{2}+1}\right)$ down to $\left[q_{0}, q_{1}\right)$ until $r_{1}$ is found. This requires computing the $\hat{Q}^{(i)}$ value for the corresponding $H^{i}(Q)$ function and checking whether $q_{i} \leqslant \tilde{Q}^{(i)}<q_{i+1}$. Recall from Corollary 1 that $\tilde{Q}^{(i)} \leqslant Q^{(i)}$. Therefore, in finding the largest realizable $\tilde{Q}^{(i)}$, if $Q^{(i)}<q_{i}$ holds for some $i$, then this implies $\tilde{Q}^{(i)}<q_{i}$, and hence, $\tilde{Q}^{(i)}$ can not be realizable. This observation may shorten the time to find $r_{1}$.

Notice that Problem $\mathscr{U} \mathscr{P} \mathscr{B} \mathscr{I}$ defined in Corollary 4 is solved only for the price intervals ranging from $r_{1}+1$ to $r_{2}$. Therefore, the result presented earlier in Proposition 1 applies here as a functional characteristic and will be used next to obtain a closed form expression for the solution of Problem $\mathscr{U P \mathscr { P } \mathscr { I }}$ in Corollary 4

Proposition 2. For $i$ s.t. $r_{1}+1 \leqslant i \leqslant r_{2}$, the solution to Problem $\mathscr{U P \mathscr { B } \mathscr { I }}$ (i.e., $Q_{i}^{*}$ ) is as follows:

- If $q_{i} \geqslant Q^{(i)}$, then $Q_{i}^{*}=q_{i}$.

- If $q_{i}<Q^{(i)}$, and

$$
\begin{aligned}
& \text { - If } q_{i+1} \geqslant Q^{(i)} \text {, then } Q_{i}^{*}=\min \left\{\left\lceil\frac{q_{i}}{P}\right\rceil P, Q^{(i)}\right\} \text {. } \\
& \text { - If } q_{i+1}<Q^{(i)} \text {, then } Q_{i}^{*}=\min \left\{\left\lceil\frac{q_{i}}{P}\right\rceil P, \lim _{Q \rightarrow q_{i+1}^{-}}\right\} \text {. }
\end{aligned}
$$

\section{An application to the newsboy problem}

In this section, we will consider the ordering decision of a company that operates under the conditions of the classical Newsboy Problem and faces an all-units quantity discount schedule and trucking costs, as in Expression (3). If the quantity ordered at the beginning of the single-period is more than the demand, excess items are salvaged at $\$ v /$ unit. If it is less than the demand, then there is a $\$ b /$ unit loss of goodwill cost. The retail price is fixed, and it is $\$ r /$ unit. Denoting the random demand amount by $X$ and its probability density function by $f(x)$, the expected profit of the newsboy is given by 
$\Pi_{b}(Q)=(r-v) \mu-(c(Q)-v) Q+(r+b-v) \int_{Q}^{\infty}(Q-x) f(x) d x-\left\lceil\frac{Q}{P}\right\rceil R$,

where $\mu$ is the expected value of demand. Here, the wholesale price function is given by an all-units discount structure, as in Expression (2). For fixed price level $c_{i}$, where $0 \leqslant i \leqslant n$, the expected profit excluding the truck costs is

$G\left(Q, c_{i}\right)=(r-v) \mu-\left(c_{i}-v\right) Q+(r+b-v) \int_{Q}^{\infty}(Q-x) f(x) d x$.

Note that the above expression is strictly concave in $Q$ with a unique maximizer at $Q^{(i)}$ that satisfies

$F\left(Q^{(i)}\right)=\frac{r+b-c_{i}}{r+b-v}$

where $F($.$) is the distribution function of demand.$

It can be easily shown that Expressions (6) and (7) satisfy assumptions (A1), (A2), and (A3) stated in Section 2. Therefore, the problem of maximizing $\Pi_{b}(Q)$ over $Q \geqslant 0$ can be solved using the algorithm in Corollary 4. Below, we present some examples of the Newsboy Problem to illustrate the application of the model introduced in Section 2 and analyzed in Section 3.

Example 1. Consider the Newsboy Problem with the following parameter settings: $r=35, b=0, v=15, R=150, P=100$ and

$c(Q)= \begin{cases}21 & 0 \leqslant Q<650, \\ 20 & 650 \leqslant Q<701, \\ 19.9 & 701 \leqslant Q<1200 \\ 19 & Q \geqslant 1200 .\end{cases}$

Demand is exponentially distributed with rate $\lambda=0.002$.

\section{Solution:}

In this example, it turns out that the profit function is given by

$\Pi_{b}(Q)=(-c(Q)+15) Q+10000-10000 e^{-0.002 Q}-\left\lceil\frac{Q}{100}\right\rceil 150$.

Here, the strictly concave component of the profit function at price level $c_{i}$ is

$G\left(Q, c_{i}\right)=\left(-c_{i}+15\right) Q+10000-10000 e^{-0.002 Q}$.

In order to maximize $\Pi_{b}(Q)$, we first find $r_{2}$, starting from the lowest price. At the lowest price $c_{3}=19$, we have $Q^{(3)}=804.719$, which is not realizable. At the next lowest price $c_{2}=19.9$, we have $Q^{(2)}=703.248$. Since $701 \leqslant Q^{(2)}<1200, Q^{(2)}$ is realizable, and hence, $r_{2}=2$. Now, starting from $i=2$, we proceed to find the value of $r_{1}$ by checking whether $\tilde{Q}^{(i)}$ is realizable. From Corollary 1 , we have $\tilde{Q}^{(2)}=600$. Since $\tilde{Q}^{(2)}<q_{2}$, it is not realizable. Similarly, at $i=1$, we have $\tilde{Q}^{(1)}=600$. Since $\tilde{Q}^{(1)}<q_{1}$, it is also not realizable. At the next price level (i.e., $c_{0}=0$ ), we have $\tilde{Q}^{(0)}=500$. Therefore, we need to compare the profits at $\tilde{Q}^{(0)}, Q_{1}^{*}, Q_{2}^{*}$, and $q_{3}$. Now, using Proposition 2, let us find the values of $Q_{1}^{*}$ and $Q_{2}^{*}$. Note that $Q^{(1)}=693.147$, and since $q_{1}<Q^{(1)} \leqslant q_{2}$, it follows that $Q_{1}^{*}=\min \left\{\left\lceil\frac{q_{1}}{100}\right\rceil 100, Q^{(1)}\right\}=Q^{(1)}=693.147$. Similarly, it turns out that $Q_{2}^{*}=Q^{(2)}=703.248$. Computing the profits at $\tilde{Q}^{(0)}, Q_{1}^{*}, Q_{2}^{*}$, and $q_{3}$, we have $\Pi_{b}\left(\tilde{Q}^{(0)}\right)=2571.21, \Pi_{b}\left(Q_{1}^{*}\right)=2984.264, \Pi_{b}\left(Q_{2}^{*}\right)=$ 2904.082, and $\Pi_{b}\left(q_{3}\right)=2492.82$. Therefore, $Q^{*}=Q_{1}^{*}=693.147$.

The above example can also be used to illustrate the magnitude of savings that can be achieved by considering transportation costs in inventory replenishment decisions. If transportation costs were ignored in decision making, the optimal order quantity would be 1200 , with a resulting expected profit of 2492.82 money units.
However, when transportation costs are considered, the optimal order quantity is 693.147, as found in the solution to Example 1. This corresponds to a $19.7 \%\left(\frac{2984.264-2492.82}{2492.82} \times 100 \%\right)$ savings. Notice that, even if an all-units discount schedule encourages the buyer to order more, savings that are inherent in transportation are realized through a lesser order quantity.

It is important to note that, although the replenishment problem in this study is posed in the context of a single echelon setting, the general model and its solution may apply to several other settings, including multi-echelon inventory problems. As an example, consider the joint replenishment decisions of a buyer and a vendor in a Newsboy setting, in which the vendor faces an all-units discount schedule, and either the vendor or the buyer has stepwise freight costs. Specifically, consider the following two scenarios in which $p(Q)$ is the vendor's procurement price given by an all-units quantity discount schedule with multiple breakpoints:

- The buyer's expected profits are as in Expression (5) with $c(Q)=c$. The vendor's profits are given by $(c-p(Q)) Q$.

- The buyer's expected profits are as in the classical Newboy Model, and the vendor's profits are given by $(c-p(Q)) Q-\left\lceil\frac{Q}{P}\right\rceil R$.

Under the above two scenarios, the expected total profit function has a structure that includes a piecewise strictly concave component reduced by stepwise freight costs, as in Expression (3). The next numerical problem exemplifies the first scenario.

Example 2. Consider a buyer-vendor system operating under the conditions of the Newsboy Problem. The buyer's unit purchasing cost, retail price, shortage cost and salvage value are $c=21, r=25, b=13, v=8$, respectively. He/she has the trucking cost parameters given by $R=70, P=100$. The vendor has the following unit purchase price schedule:

$p(Q)= \begin{cases}20 & 0 \leqslant Q<201 \\ 18 & 201 \leqslant Q<401 \\ 16 & 401 \leqslant Q<601 \\ 14 & Q \geqslant 601\end{cases}$

Demand is uniformly distributed between 400 and 600 .

\section{Solution:}

The buyer's expected profit function $\Pi_{b}(Q)$ and the vendor's expected profit function $\Pi_{v}(Q)$ are as follows:

$\Pi_{b}(Q)=77 Q-\frac{3 Q^{2}}{40}-18500-\left\lceil\frac{Q}{100}\right\rceil 70$,
$\Pi_{v}(Q)=(21-p(Q)) Q$.

Let us find the order quantity that maximizes the expected total profits of the system, which is given by

$$
\begin{aligned}
\Pi(Q) & =\Pi_{b}(Q)+\Pi_{v}(Q) \\
& =(-p(Q)+98) Q-\frac{3 Q^{2}}{40}-18500-\left\lceil\frac{Q}{100}\right\rceil 70 .
\end{aligned}
$$

Denoting the vendor's purchasing price in the ith interval by $p_{i}$, the strictly concave component of the profit function at price $p_{i}$ is

$G\left(Q, p_{i}\right)=\left(-p_{i}+98\right) Q-\frac{3 Q^{2}}{40}-18500$

We again start from the lowest price to search for $r_{2}$. At $p_{3}=14$, it turns out that $Q^{(3)}=560$, which is not realizable. At the next lowest price $p_{2}=16$, we have $401 \leqslant Q^{(2)}=546.57<601$, and therefore, $r_{2}=2$. Utilizing Corollary 1 , we also find $\tilde{Q}^{(2)}$ as 546.57 , and hence, $r_{1}=2$. Corollary 4 implies that we need to compare the expected 
system profits at $\tilde{Q}^{\left(r_{1}\right)}=546.57$ and at $q_{3}$. Since $\Pi(546.57)=$ 3493.33 and $\Pi(601)=4403.94$, we have $Q^{*}=q_{3}=601$.

Last, but not least, consider a scenario in which the vendor has capacitated production setups or trucks used inbound replenishment. The unit procurement cost for the vendor, denoted by $p$, is constant, and therefore, the vendor's profits are given by $(c-p) Q-\left\lceil\frac{Q}{P}\right\rceil R$. The buyer in this system operates under the conditions of the Newsboy Model and uses a common carrier for transportation who charges on the basis of an all-units discount schedule. Obviously, the solution to the joint replenishment decision in this scenario can again be found using the approach presented in our paper.

\section{Implications for inventory management}

Freight costs constitute a major part of the world's biggest economies. For example, according to the 17th Annual State of Logistics Report [30], U.S. business logistics costs were 9.5\% of the nominal GDP in 2005. Transportation costs, as a significant portion of logistical expenses, accounted for $6 \%$ of the nominal GDP. This implies that significant savings can be achieved through carefully planning for transportation. To this end, researchers have shown in numerous studies that transportation decisions should be made simultaneously with inventory replenishment decisions. Based on this premise, in the current study, we model and solve the replenishment problem of a company that faces all-units quantity discount and stepwise freight costs.

Inventory replenishment decisions have been mostly made without giving consideration to transportation costs. Carter and Ferrin [7] show that substantial savings can be attained by increasing the order quantity when an LTL common carrier is used. This is achieved by taking advantage of the reductions in freight rate for larger quantities. Our finding is that, in the presence of TL transportation, savings can sometimes be realized by decreasing the order quantity. This is specifically important in the presence of quantity discounts, because such discounts encourage the buyer to order more. The tendency to order more under quantity discounts may result in increased transportation costs and may not justify the use of additional trucks in the case of TL transportation.

\section{Conclusions}

This study considers a single echelon replenishment problem with all-units quantity discounts and generalized transportation costs. Quantity discounts are widely used in industry by suppliers to attract more buyers, to increase buyers' order sizes, and to take advantage of economies of scale. In fact, quantity discounts are one of the mechanisms to achieve coordination in supply chains and to share the extra savings due to coordination. A quantity discount with multiple breakpoints may prevail under the existence of multiple buyers with the purpose of price discrimination. In addition, when several alternative suppliers offer all-units discount schedules with single but different breakpoints, the wholesale price schedule that is faced by a buyer turns out to have multiple breakpoints. Our analysis accounts for both a wholesale price schedule in the form an all-units quantity discount with multiple breakpoints and stepwise freight costs.

The replenishment problem is formulated in terms of a general model with an objective function that includes a piecewise strictly concave component reduced by stepwise fixed increments. A computational solution procedure is proposed, based on several properties of the objective function. The model and its solution are later applied to the Newsboy Problem, under given cost considerations. As it is also illustrated over some examples, with the gen- eral analysis in this study, one can find solutions to several replenishment problems, including those in multi-echelon settings.

This study can be used as a first step analysis to coordination problems under the existence of many buyers with stepwise freight costs. Recall that our premise is that the all-units discount schedule is already given. A natural extension would be to consider the vendor's problem in designing a price schedule that coordinates the system comprising his/her buyers who face transportation costs and capacities.

\section{Appendix A}

\section{A.1. Proof of Property 6}

Assume that $\exists j>\left(r_{2}+1\right)$ s.t. $G\left(Q, c_{j-1}\right)$ is increasing in $Q$ over $q_{j-1} \leqslant Q<q_{j}$. Then, we should have $Q^{(j-1)} \geqslant q_{j}$. Since $Q^{(j)}>Q^{(j-1)}$, it turns out that $Q^{(j)}>q_{j}$. This implies that $Q^{(j)} \geqslant q_{j+1}$, because otherwise, if we had $q_{j}<Q^{(j)}<q_{j+1}$, then $Q^{(j)}$ would be realizable. Therefore, $G\left(Q, c_{j}\right)$ has to be increasing over $q_{j} \leqslant Q<q_{j+1}$. In a similar fashion, $G\left(Q, c_{j+1}\right), G\left(Q, c_{j+2}\right), \ldots, G\left(Q, c_{n-1}\right)$ have to be increasing over $\quad q_{j+1} \leqslant Q<q_{j+2}, q_{j+2} \leqslant Q<q_{j+3}, \ldots, q_{n-1} \leqslant Q<q_{n}$, respectively, and, we should have $Q^{(j+1)} \geqslant q_{j+2}, Q^{(j+2)} \geqslant$ $q_{j+3}, \ldots, Q^{(n-1)} \geqslant q_{n}$. Since $Q^{(n)}>Q^{(n-1)}$, this would imply that $Q^{(n)}>q_{n}$. However, this contradicts with the fact that $Q^{\left(r_{2}\right)}$ is the largest realizable maximizer of $G\left(Q, c_{i}\right)$ 's.

\section{A.2. Proof of Property 7}

Since $Q^{\left(r_{2}\right)}$ is the largest realizable maximizer of $G\left(Q, c_{i}\right)$ s, it follows from Property 6 that $G\left(Q, c_{k-1}\right)<G\left(q_{k-1}, c_{k-1}\right)$ where $q_{k-1}<Q<q_{k}, \forall k$ s.t. $k>r_{2}+1$. Since $\left\lceil\frac{Q}{P}\right\rceil R \geqslant\left\lceil\frac{q_{k-1}}{P}\right\rceil R$, it turns out that $G\left(Q, c_{k-1}\right)-\left\lceil\frac{Q}{P}\right\rceil R<G\left(q_{k-1}, c_{k-1}\right)-\left\lceil\frac{q_{k-1}}{P}\right\rceil R$, and hence, $H^{k-1}(Q)<H^{k-1}\left(q_{k-1}\right)$. For $Q \in\left[q_{k-1}, q_{k}\right)$, we have $\mathscr{H}(Q)=H^{k-1}(Q)$ and $\mathscr{H}\left(q_{k-1}\right)=H^{k-1}\left(q_{k-1}\right)$. Therefore, $\mathscr{H}(Q)<\mathscr{H}\left(q_{k-1}\right)$ where $q_{k-1}<Q<q_{k}, \forall k$ s.t. $k>r_{2}+1$.

\section{A.3. Proof of Property 8}

Since $G\left(\tilde{Q}^{(i)}, c_{i}\right)<G\left(\tilde{Q}^{(i)}, c_{i+1}\right)$, it follows that $G\left(\tilde{Q}^{(i)}, c_{i}\right)-$ $\left\lceil\frac{\tilde{Q}^{(i)}}{P}\right] R<G\left(\tilde{Q}^{(i)}, c_{i+1}\right)-\left\lceil\frac{\tilde{Q}^{(i)}}{P}\right] R$, and hence, $H^{i}\left(\tilde{Q}^{(i)}\right)<H^{i+1}\left(\tilde{Q}^{(i)}\right)$. We have $H^{i+1}\left(\tilde{Q}^{(i+1)}\right) \geqslant H^{i+1}\left(\tilde{Q}^{(i)}\right)$, therefore, $H^{i+1}\left(\tilde{Q}^{(i+1)}\right)>H^{i}\left(\tilde{Q}^{(i)}\right)$.

\section{A.4. Proof of Property 9}

Let us assume $\exists j, 0 \leqslant j \leqslant n-1$, such that $\tilde{Q}^{(j)}>\tilde{Q}^{(j+1)}$. We have from Corollary 1 that $\tilde{Q}^{(j)} \leqslant Q^{(j)}$. Since $Q^{(j+1)}>Q^{(j)}$, it follows that $Q^{(j+1)}>\tilde{Q}^{(j+1)}$. This implies that $\tilde{Q}^{(j+1)}=k P$ for some $k \in\{0,1,2, \ldots\}$, and therefore, either one of the following cases should hold.

Case 1: $G\left((k+1) P, c_{j+1}\right)-G\left(k P, c_{j+1}\right) \leqslant R$ where $(k+1) P \leqslant Q^{(j+1)}$.

Since $G\left((k+1) P, c_{j}\right)-G\left(k P, c_{j}\right)<G\left((k+1) P, c_{j+1}\right)-G\left(k P, c_{j+1}\right)$, it follows that $G\left((k+1) P, c_{j}\right)-G\left(k P, c_{j}\right)<R$. Therefore, we should have $\tilde{Q}^{(j)} \leqslant k P$, which contradicts with $\tilde{Q}^{(j)}>\tilde{Q}^{(j+1)}$.

Case 2: $\tilde{Q}^{(j+1)}=l P$ where $l=\left\lceil\frac{Q^{(j+1)}}{P}\right\rceil-1$.

Since $H^{j+1}(l P) \geqslant H^{j+1}\left(Q^{(j+1)}\right)$, it follows that $G\left(Q^{(j+1)}, c_{j+1}\right)-$ $G\left(l P, c_{j+1}\right) \leqslant R$. Using the fact that $G\left(Q^{(j+1)}, c_{j+1}\right)>G\left(Q^{(j)}, c_{j+1}\right)$, we have $G\left(Q^{(j)}, c_{j+1}\right)-G\left(l P, c_{j+1}\right)<R$. Now, since $\tilde{Q}^{(j+1)}=l P<\tilde{Q}^{(j)}$ and $\tilde{Q}^{(j)} \leqslant Q^{(j)}<Q^{(j+1)}$, it turns out that $I P<\tilde{Q}^{(j)}<Q^{(j+1)}$, and hence, $l=\left\lceil\frac{\hat{Q}^{(j)}}{P}\right]-1$. Since $G\left(Q^{(j)}, c_{j+1}\right)-G\left(l P, c_{j+1}\right)<R$, we also have $G\left(Q^{(j)}, c_{j}\right)-G\left(l P, c_{j}\right)<R$. Therefore, $G\left(Q^{(j)}, c_{j}\right)-(l+1) R<G\left(l P, c_{j}\right)$ $-l R$, and hence, $H^{j}\left(Q^{(j)}\right)<H^{j}(l P)$. This implies that $\tilde{Q}^{(j)} \leqslant l P=\tilde{Q}^{(j+1)}$, which contradicts our assumption. 


\section{A.5. Proof of Property 10}

Assume that there exists no realizable $\tilde{Q}^{(i)}$. Then, $\tilde{Q}^{(0)} \geqslant q_{1}$. Since $\tilde{Q}^{(1)} \geqslant \tilde{Q}^{(0)}$, then $\tilde{Q}^{(1)} \geqslant q_{2}$, because otherwise, if $q_{1} \leqslant \tilde{Q}^{(1)}<q_{2}$, then $\tilde{Q}^{(1)}$ would be realizable. Since $\tilde{Q}^{(2)} \geqslant \tilde{Q}^{(1)}$, then $\tilde{Q}^{(2)} \geqslant q_{3}$, because otherwise, if $q_{2} \leqslant \tilde{Q}^{(2)}<q_{3}$, then $\tilde{Q}^{(2)}$ would be realizable. Continuing in this fashion, we have $\tilde{Q}^{(n-1)} \geqslant q_{n}$. Since $\tilde{Q}^{(n)} \geqslant \tilde{Q}^{(n-1)}$, then $\tilde{Q}^{(n)} \geqslant q_{n}$. However, this would make $\tilde{Q}^{(n)}$ realizable, which is a contradiction.

\section{A.6. Proof of Corollary 3}

Corollaries 1 and 2 imply that $\tilde{Q}^{(k)}<q_{k}, \forall k \geqslant\left(r_{2}+1\right)$. In other words, $\tilde{Q}^{(k)}$ values are not realizable $\forall k \geqslant\left(r_{2}+1\right)$. This implies that $r_{1} \leqslant r_{2}$ and $\tilde{Q}^{\left(r_{1}\right)} \leqslant Q^{\left(r_{2}\right)}$.

\section{A.7. Proof of Proposition 1}

Assume that $\exists k, r_{1}<k<r_{2}$ such that $\tilde{Q}^{(k)} \geqslant q_{k}$. Then, we should have $\tilde{Q}^{(k)} \geqslant q_{k+1}$, because otherwise, if we had $q_{k} \leqslant \tilde{Q}^{(k)}<q_{k+1}$, then $\tilde{Q}^{(k)}$ would be realizable. Since $\tilde{Q}^{(k+1)} \geqslant \tilde{Q}^{(k)}$, we have $\tilde{Q}^{(k+1)} \geqslant q_{k+1}$. This implies that $\tilde{Q}^{(k+1)} \geqslant q_{k+2}$. Continuing in this fashion, we have $\tilde{Q}^{\left(r_{2}-1\right)} \geqslant q_{r_{2}-1}$, which leads to $\tilde{Q}^{\left(r_{2}-1\right)} \geqslant q_{r_{2}}$. Since $\tilde{Q}^{\left(r_{2}\right)} \geqslant \tilde{Q}^{\left(r_{2}-1\right)}$, we have $\tilde{Q}^{\left(r_{2}\right)} \geqslant q_{r_{2}}$. We know that $Q^{\left(r_{2}\right)}$ is realizable, therefore, $q_{r_{2}} \leqslant Q^{\left(r_{2}\right)}<q_{r_{2}+1}$. Also, we have from Corollary 1 that $\tilde{Q}^{\left(r_{2}\right)} \leqslant Q^{\left(r_{2}\right)}$. Hence, we should have $q_{r_{2}} \leqslant \tilde{Q}^{\left(r_{2}\right)}<q_{r_{2}+1}$. However, this contradicts the fact that $\tilde{Q}^{\left(r_{1}\right)}$ is the largest realizable maximizer of $H^{i}(Q)$ s.

\section{A.8. Proof of Proposition 2}

For the first case (i.e., $q_{i} \geqslant Q^{(i)}$ ) the result follows from Property 1. We analyze the second case (i.e., $q_{i}<Q^{(i)}$ ) in two subcases.

Subcase I: $q_{i+1} \geqslant Q^{(i)}$.

Observe that in this subcase $Q^{(i)}$ is realizable, and either $\left\lceil\frac{q_{i}}{P}\right\rceil=\left\lceil\frac{Q^{(i)}}{P}\right\rceil$ or $\left\lceil\frac{q_{i}}{P}\right\rceil<\left\lceil\frac{Q^{(i)}}{P}\right\rceil$ holds. If $\left\lceil\frac{q_{i}}{P}\right\rceil=\left\lceil\frac{Q^{(i)}}{P}\right\rceil$, since $Q^{(i)}$ is the maximizer of $G\left(Q, c_{i}\right)$ we have $Q_{i}^{*}=Q^{(i)}$. Note that $\left\lceil\frac{q_{i}}{P}\right\rceil=\left\lceil\frac{Q^{(i)}}{P}\right\rceil$ implies $\left\lceil\frac{q_{i}}{P}\right\rceil P \geqslant Q^{(i)}$, and hence $\min \left\{\left\lceil\frac{q_{i}}{P}\right\rceil P, Q^{(i)}\right\}=Q^{(i)}$. If $\left\lceil\frac{q_{i}}{P}\right\rceil<\left\lceil\frac{Q^{(i)}}{P}\right\rceil$, utilizing Properties 2 and 3 under the fact that $\tilde{Q}^{(i)}<q_{i}$, we conclude $Q_{i}^{*}=\left\lceil\frac{q_{i}}{P}\right\rceil P$. Similarly, $\left\lceil\frac{q_{i}}{P}\right\rceil<\left\lceil\frac{Q^{(i)}}{P}\right\rceil$ implies $\left\lceil\frac{q_{i}}{P}\right\rceil P<Q^{(i)}$, and hence $\min \left\{\left\lceil\frac{q_{i}}{P}\right\rceil P, Q^{(i)}\right\}=\left\lceil\frac{q_{i}}{P}\right\rceil P$.

Subcase II: $q_{i+1}<Q^{(i)}$.

Observe that in this subcase $Q^{(i)}$ is not realizable, and either $\left\lceil\frac{q_{i}}{P}\right\rceil=\left\lceil\frac{q_{i+1}}{P}\right\rceil$ or $\left\lceil\frac{q_{i}}{P}\right\rceil<\left\lceil\frac{q_{i+1}}{P}\right\rceil$ holds. If $\left\lceil\frac{q_{i}}{P}\right\rceil=\left\lceil\frac{q_{i+1}}{P}\right\rceil$, Property 2 implies $Q_{i}^{*}=\lim _{Q \rightarrow q_{i+1}^{-}}$. Note that when $\left\lceil\frac{q_{i}}{P}\right\rceil=\left\lceil\frac{q_{i+1}}{P}\right\rceil$, we have $\left\lceil\frac{q_{i}}{P}\right\rceil P>\lim _{Q \rightarrow q_{i+1}^{-}}$, and hence $\min \left\{\left\lceil\frac{q_{i}}{P}\right\rceil P, \lim _{Q \rightarrow q_{i+1}^{-}}\right\}=\lim _{Q \rightarrow q_{i+1}^{-}}$. If $\left\lceil\frac{q_{i}}{P}\right\rceil<\left\lceil\frac{q_{i+1}}{P}\right\rceil$, utilizing Properties 2 and 3 again under the fact that $\hat{Q}^{(i)}<q_{i}$ and $q_{i+1}<Q^{(i)}$, we conclude $Q_{i}^{*}=\left\lceil\frac{q_{i}}{P}\right\rceil P$. Similarly, $\quad\left\lceil\frac{q_{i}}{P}\right\rceil<\left\lceil\frac{q_{i+1}}{P}\right\rceil$ implies $\left\lceil\frac{q_{i}}{P}\right\rceil P<\lim _{Q \rightarrow q_{i+1}^{-}}$, and hence $\min \left\{\left\lceil\frac{q_{i}}{P}\right\rceil P, \lim _{Q \rightarrow q_{i+1}^{-}}\right\}=\left\lceil\frac{q_{i}}{P}\right\rceil P$.

\section{References}

[1] P.L. Abad, Determining optimal selling price and lot size when the supplier offers all-unit quantity discounts, Decision Sciences 19 (1988) 622-634.

[2] F.J. Arcelus, G. Srinivasan, Discount strategies for one-time only sales, IIE Transactions 27 (1995) 618-624.

[3] D.C. Aucamp, Nonlinear freight costs in the EOQ model, European Journal of Operational Research 9 (1982) 61-63.

[4] A. Banerjee, On a quantity discount pricing model to increase vendor profits, Management Science 32 (1986) 1513-1517.

[5] W.C. Benton, S. Park, A classification of literature on determining the lot size under quantity discounts, European Journal of Operational Research 92 (1996) 219-238.

[6] T.H. Burwell, D.S. Dave, K.E. Fitzpatrick, M.R. Roy, Economic lot size model for price-dependent demand under quantity and freight discounts, International Journal of Production Economics 48 (1997) 141-155.

[7] J.R. Carter, B.G. Ferrin, Transportation costs and inventory management: Why transportation costs matter, Production and Inventory Management Journal 37 (1996) 58-62.

[8] S. Cetinkaya, C.-Y. Lee, Optimal outbound dispatch policies modeling inventory and cargo capacity, Naval Research Logistics 49 (2002) 531-556.

[9] L.M.A. Chan, A. Muriel, Z.-J. Shen, D. Simchi-Levi, C.-P. Teo, Effective zero inventory ordering polices for the single-warehouse multi-retailer problem with piecewise linear cost structures, Management Science 48 (2002) 14461460.

[10] C.J. Corbett, X. de Groote, A supplier's optimal quantity discount policy under asymmetric information, Management Science 46 (2000) 444-450.

[11] C. Das, A unified approach to the price-break economic order quantity (EOQ) problem, Decision Sciences 15 (1984) 350-358.

[12] K. Ertogral, M. Darwish, M. Darwish, Production and shipment lot sizing in a vendor-buyer supply chain with transportation cost, European Journal of Operational Research 176 (2007) 1592-1606.

[13] G. Hadley, T.M. Whitin, Analysis of Inventory Systems, Prentice Hall Englewood Cliffs, NJ, 1963

[14] M.A. Hoque, S.K. Goyal, An optimal policy for single-vendor single-buyer integrated production-inventory system with capacity constraint of transport equipment, International Journal of Production Economics 65 (2000) 305-315.

[15] H. Hwang, D.H. Moon, S.W. Shinn, An EOQ model with quantity discounts for both purchasing price and freight cost, Computers and Operations Research 17 (1990) 73-78.

[16] T.W. Knowles, P. Pantumsinchai, All-units discounts for standard container sizes, Decision Sciences 19 (1988) 848-857.

[17] R. Lal, R. Staelin, An approach for developing an optimal discount pricing policy, Management Science 30 (1984) 1524-1539.

[18] C.-Y. Lee, The economic order quantity for freight discount costs, IIE Transactions 18 (1986) 318-320.

[19] C.-Y. Lee, A solution to the multiple set-up problem with dynamic demand, IIE Transactions 21 (1989) 266-270.

[20] H.L. Lee, M.J. Rosenblatt, A generalized quantity discount pricing model to increase supplier's profits, Management Science 32 (1986) 1177-1185.

[21] J.P. Monahan, A quantity discount pricing model to increase vendor profits, Management Science 30 (1984) 720-726.

[22] C. Munson, M.J. Rosenblatt, Theories and realities of quantity discounts: An exploratory study, Production and Operations Management 7 (1998) 352-369.

[23] R.M. Russell, L.J. Krajewski, Optimal purchase and transportation cost lot sizing for a single item, Decision Sciences 22 (1991) 940-952.

[24] S.W. Shinn, H. Hwang, S.S. Park, Joint price and lot size determination under conditions of permissible delay in payments and quantity discounts for freight cost, European Journal of Operational Research 91 (1996) 528-542.

[25] R.J. Tersine, S. Barman, Optimal lot sizes for unit and shipping discount situations, IIE Transactions 26 (1994) 97-101.

[26] R.J. Tersine, S. Barman, Economic purchasing strategies for temporary price discounts, European Journal of Operational Research 80 (1995) 328-343.

[27] A. Toptal, S. Çetinkaya, C.-Y. Lee, The buyer-vendor coordination problem: Modeling inbound and outbound cargo capacity and costs, IIE Transactions on Logistics and Scheduling 35 (2003) 987-1002.

[28] A. Toptal, S. Çetinkaya, Contractual agreements for coordination and vendormanaged delivery under explicit transportation considerations, Naval Research Logistics 53 (2006) 1-21.

[29] Z.K. Weng, Channel coordination and quantity discounts, Management Science 41 (1995) 1509-1522.

[30] R. Wilson, 17th Annual State of Logistics Report, 2006, <http://www.loginsti tute.ca/research/index_sub.php?id = 67\&level $=3>$ (accessed February 2008). 